\title{
Formation of Superclusters from Metallic Clusters
}

\author{
By Hans-Gerhard Fritsche', Hans Müller' and Benjamin Fehrensen² \\ ' Institut für Physikalische Chemie der Chemisch-Geowissenschaftlichen Fakultät \\ der Friedrich-Schiller-Universität, Am Steiger 3, D-07743 Jena, Germany \\ 2 ETH Zürich, Institut für Physikalische Chemie, Zentrum, \\ Universitätsstraße 22, CH-8092 Zürich, Switzerland
}

(Received August 23, 1996)

\section{Mesoscopic particles / Supercluster / Size effects / Surface dependent properties / Embedded atom approximation}

Schmid et al. [3] reported on the possibility to build up experimentally microcrystalline modifications of metals with icosahedral clusters $M_{13}$ as the basic units of the structure. We theoretically investigate two possibilities to construct such superclusters: a) a formation of shell-like superclusters, and b) a generation of superclusters from superclusters.

The numerically obtained results agree very well with many experimental data of the new metallic modification. They explain the growth of superclusters by a simple analytical model. Size effects of some mechanical properties of such superclusters can be predicted by analytical relations.

Schmid et al. [3] berichteten von der experimentellen Erzeugung mikrokristalliner Modifikationen von Metallen mit ikosaedrischen Clustern $M_{13}$ als strukturellen Grundeinheiten. Wir untersuchten daraufhin mit theoretischen Methoden zwei Wege der Konstruktion solcher Supercluster: a) die Bildung schalenförmiger Supercluster und b) die Bildung von Superclustern aus Superclustern.

Die numerisch gewonnenen Ergebnisse stimmen recht gut mit experimentellen Daten der neuen metallischen Modifikation überein. Auf ihrer Grundlage ergibt sich ein analytisches Modell des Wachstums von Superclustern, welches die Vorhersage der Größenabhängigkeit mechanischer Eigenschaften von Superclustern erlaubt.

\section{Introduction}

The high stability of transition metal cluster compounds with a core of geometrically closed shells of metal atoms is well known [1,2]. Such compounds can be used to produce ligand-free $M_{13}$ clusters by degradation of the ligands and the outer-shell metal atoms [3]. These naked icosahedral 
$M_{13}$ clusters do not decompose into smaller particles (as supposed [4]), but they aggregate to superclusters. Two lines of the formation of superclusters have been found experimentally:

1) the generation of superclusters of one or two full shells of clusters $M_{13}[5,6]$

$$
\begin{aligned}
& 13 M_{13} \rightarrow\left(M_{1.3}\right)_{1.3} \\
& 55 M_{13} \rightarrow\left(M_{1.3}\right)_{55}
\end{aligned}
$$

and

2) the generation of superclusters from clusters/superclusters $[3,6,9]$

$$
\begin{aligned}
& 13 M_{13} \rightarrow\left(M_{13}\right)_{13} \\
& 13\left(M_{13}\right)_{1.3} \rightarrow\left(\left(M_{13}\right)_{13}\right)_{1.3} \\
& 13\left(\left(M_{13}\right)_{1.3}\right)_{1.3} \rightarrow\left(\left(\left(M_{13}\right)_{13}\right)_{13}\right)_{1.3}
\end{aligned}
$$

Here, only the first steps of both lines are identical.

Some years ago we derived from a simple jellium model within an iterative version this aufbauprinciple as a disign principle for new metals $[7,8]$.

The formation of superclusters from superclusters can be repeated stepwise (Fig. 1). The resulting microcrystalline products of the metals $\mathrm{Au}, \mathrm{Co}$, $\mathrm{Pt}, \mathrm{Rh}$, and $\mathrm{Ru}$ have been structurally analized by various experimental techniques (time of flight secondary ion mass spectrometry, X-ray powder diffraction, Mössbauer spectrometry). It has been found, that the new metallic modification is stable at low temperatures. At higher temperatures (for $\left(\left(\mathrm{Au}_{13}\right)_{1.3}\right)_{13}$ at $\left.700-800 \mathrm{~K}\right)$, it is transferred into the structure of normal bulk metal [9].

It can be expected, that this new microcrystalline modification has properties different from that ones of the corresponding bulk metal. Some of them will be investigated in this paper theoretically by use of the Embedded Atom Method (EAM).

\section{The embedded atom method (EAM)}

The embedded atom method has been described in detail in many papers [10-13]. The method was successfully applied to many problems of metals (clusters and bulk). For a review, see [13]. Here, we only report on some few aspects of the method.

We start with an approximation of the total potential energy of interaction by additive attractive embedding energies, $F_{i}$, and pairwise additive energies of repulsion, $\Phi_{i j}$, of the $N$ atoms of the cluster $M_{N}$ :

$$
E_{\mathrm{tot}}=\sum_{i=1}^{N} F_{i}\left(\varrho_{h . i}\right)+\sum_{i<j=1}^{N} \Phi_{i j}\left(R_{i j}\right) .
$$




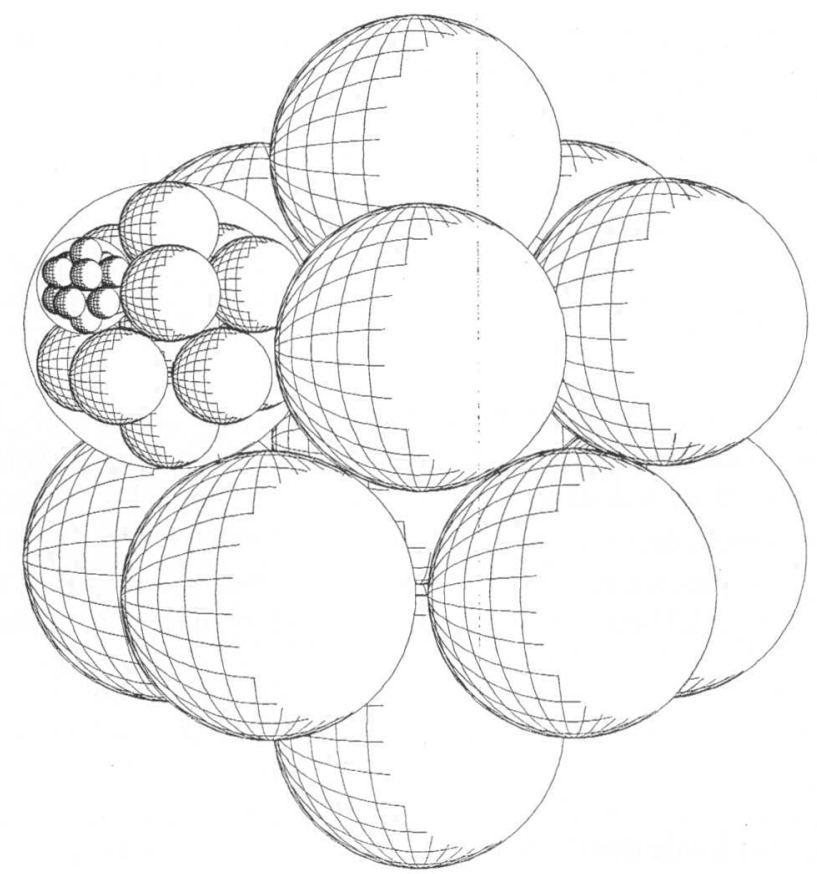

Fig. 1. Supercluster $\left(\left(M_{13}\right)_{13}\right)_{13}$ : generation line 2, product of Eq. (2b).

In this expression, $\varrho_{h, i}$ is the host electron density at atom $i$ due to the remaining atoms, $F_{i}(\varrho)$ is the energy to embed atom $i$ into the background electron density $\varrho$, and $\Phi_{i j}\left(R_{i j}\right)$ is the core-core pair repulsion between atoms $i$ and $j$ separated by the distance $R_{i j}$. The host electron density is approximated by the superposition of densities of valence electrons

$$
\varrho_{h, i}=\sum_{j(\neq i)=1}^{N} \varrho^{j}\left(R_{i j}\right),
$$

where $\varrho^{j}\left(R_{i j}\right)$ is the electron density at atom $i$ contributed by atom $j$. The atomic densities will be taken from the tables of Clementi et al. [14], and McLean et al. [15]. Values of the functions $F$ and $\Phi$ can be calculated from the formal definitions of these quantities within the density-functional framework [16], but it is usual to establish $F$ and $\Phi$ empirically from physical properties of solids (lattice constant, elastic constants, cohesive energy, and vacancy-formation energy) $[10,12]$.

Numerical calculations have been carried out here for $M=\mathrm{Li}, \mathrm{Ni}$, and $\mathrm{Au}$. We used the EAM-parametrization of Zhang et al. (lithium, [17]), and of Voter et al. (nickel, [18]). For gold, we determined new EAM-parameters [19]. 
Table 1. Equilibrium data of icosahedral clusters $M_{13}(M=\mathrm{Li}, \mathrm{Ni}, \mathrm{Au})$ optimized by EAM calculations: $r_{1}=$ shortest interatomic distance, $E / 13=$ binding energy per atom. $r_{\text {cut }}=$ cut-off radius of EAM-interactions.

\begin{tabular}{lllll}
\hline$M_{13}$ & $r_{\text {cut }}[\AA]$ & $r_{1}[\AA]$ & $-E / 13[\mathrm{eV}]$ & $r_{\text {cut }} / r_{1}$ \\
\hline $\mathrm{Li}_{13}$ & 4.9629 & 2.823 & 1.279 & 1.758 \\
$\mathrm{Ni}_{133}$ & 4.7895 & 2.382 & 3.034 & 2.011 \\
$\mathrm{Au}_{13}$ & 3.4739 & 2.630 & 3.101 & 1.321 \\
\hline
\end{tabular}

The contributions to the energy of interaction of atoms separated from each other more than a distance $r_{\text {cut }}$ are very small (contributions to the host electron density $\varrho_{h, i}$ as well as contributions to the core-core repulsion $\Phi_{i j}$ ). Usually, they are neglected. (Their neglection is incorporated into the determination of the EAM-parameters by the adaption of experimental data of solids.)

\section{Results}

\subsection{Icosahedral basic clusters $M_{13}$}

First, we optimized the basic icosahedral clusters $M_{13}$. The results are given in Table 1. The shortest interatomic distances, $r_{1}$, and the binding energies per atom, $E / 13$, are qualitatively correct (between the experimental values of bulk and that ones of the diatomic molecule). For detailed discussion, see [19]. The last column of Table 1 shows the maximum range of interatomic interactions in units of the shortest interatomic distance, $r_{1}$, of the icosahedral.

\subsection{The dimeric supercluster $\left(M_{13}\right)_{2}$}

It has been verified, that two icosahedral clusters $M_{13}$ at their equilibrium distance, $r_{2}$, have a rigid structure to a high degree of accuracy [20].

As an example, Fig. 2 shows the cluster-cluster interaction energy, $\Delta E(L=2)=E(2 \cdot 13)-2 \cdot E(13)$, of the dimeric supercluster $\left(\mathrm{Au}_{13}\right)_{2}$ in dependence on the distance between the both clusters, $r_{2}$. The values at the equilibrium are: $\Delta E=-0.45 \mathrm{eV}, r_{2}=7.89 \AA$. In harmonic approximation, the frequency of the relative oscillation of the both clusters is $v=$ $3.8 \cdot 10^{11} \mathrm{~Hz}$, the corresponding wave length is $\lambda=0.08 \mathrm{~cm}$.

\subsection{Formation of bigger superclusters}

Now, we study the formation of superclusters $\left(M_{13}\right)_{L}$ from $L$ rigid icosahedral clusters $M_{13}$. 


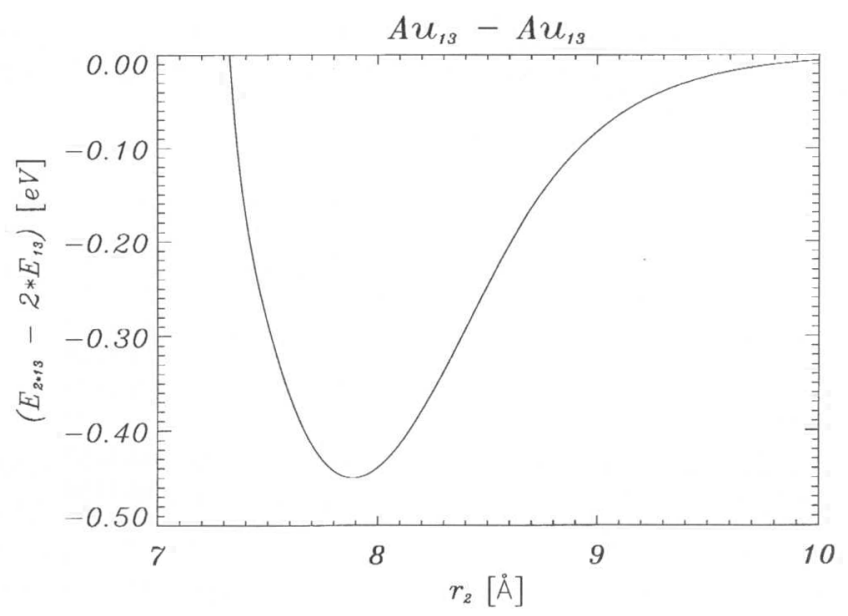

Fig. 2. Cluster-cluster interaction energy of the dimeric supercluster $\left(\mathrm{Au}_{13}\right)_{2}$.

In fact, the energy of the cluster-cluster interaction

$$
\Delta E=E(L \cdot 13)-L \cdot E(13)
$$

will depend on the relative orientation of the clusters, but it will be shown in section 3.3.1, that changes of this orientation will influence the quantity $\Delta E$ only by a constant factor. Interesting qualitative results can be obtained by the only optimization of the distance $r_{2}$ between two neighbouring clusters of the supercluster, too. In this sense, we define here the structure of the supercluster to be fixed. All clusters $M_{13}$ inside of the supercluster have the same orientation of their local $z$-axis.

\subsubsection{Generation line 1}

As an example of the generation line 1, we report on the formation of icosahedral superclusters $\left(\mathrm{Li}_{13}\right)_{L}$ consisting of $n$ closed icosahedral shells of icosahedral cluster units $\mathrm{Li}_{13}$. Investigations of other structures of superclusters (linear, squared, simple cubic, body-centered cubic, face-centered cubic) gave similar results. In this notation, the centres of the basic clusters $\mathrm{Li}_{13}$ define the polyhedron of the supercluster. We studied superclusters of $n=1,2, \ldots, 8$ shells with $L=13,55, \ldots, 2057$ cluster units or $N=$ $169,715, \ldots, 26741$ atoms and determined the optimum shortest distance $r_{2}$ of two neighbouring clusters $\mathrm{Li}_{13}$ for each supercluster. The results are given in Table 2 and in Fig. 3. 
Table 2. Equilibrium data of shell-like icosahedral superclusters $\left(\mathrm{Li}_{13}\right)_{L}$ (generation line 1): $r_{2}=$ shortest intercluster pair distance, $\Delta E / L=$ cluster-cluster interaction energy per cluster unit, $\triangle E /\left(L \cdot C N_{1}\right)=$ interaction energy of one pair of clusters of the supercluster.

\begin{tabular}{lrlll}
\hline$n$ & $L$ & $r_{2}[\AA]$ & $-\Delta E / L[\mathrm{eV}]$ & $-\Delta E /\left(L \cdot C N_{1}\right)[\mathrm{eV}]$ \\
\hline 1 & 13 & 8.3199 & 0.3906 & 0.1209 \\
2 & 55 & 8.3814 & 0.4969 & 0.1168 \\
3 & 147 & 8.3969 & 0.5458 & 0.1153 \\
4 & 309 & 8.4057 & 0.5737 & 0.1145 \\
5 & 561 & 8.4113 & 0.5916 & 0.1140 \\
6 & 923 & 8.4152 & 0.6040 & 0.1137 \\
7 & 1415 & 8.4181 & 0.6132 & 0.1135 \\
8 & 2057 & 8.4203 & 0.6203 & 0.1134 \\
\hline
\end{tabular}

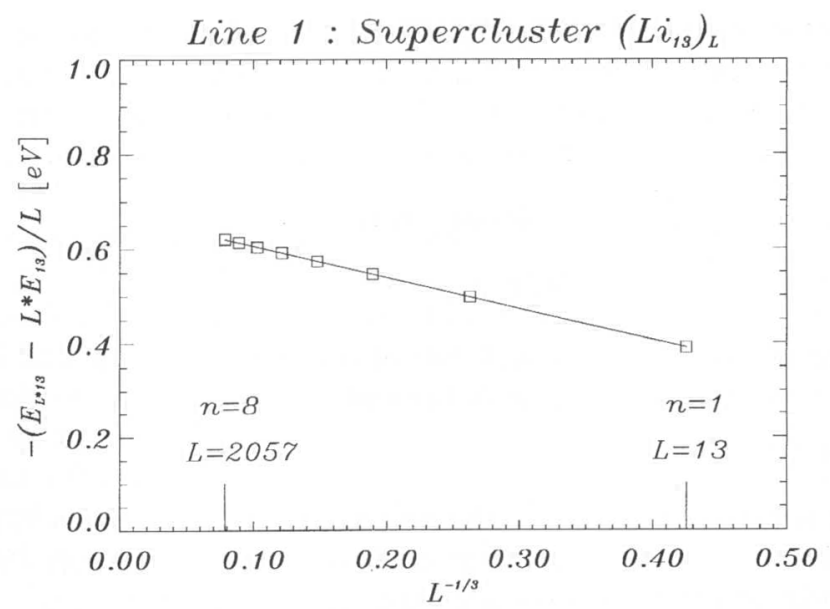

Fig. 3. Size effect of the cohesive energy, $\Delta E / L$, of icosahedral superclusters $\left(\operatorname{Li}_{13}\right)_{\text {, with }}$ $n=1,2, \ldots, 8$ closed shells (generation line 1 ).

We see:

1) The shortest intercluster distance, $r_{2}$, is nearly constant.

2) The cluster-cluster interaction energy per cluster, $\Delta E / L$, increases with the size of the supercluster. Fig. 3 shows, that the size-effect of $\Delta E / L$ can be described by the equation

$$
\Delta E / L \approx a+b \cdot L^{-1 / 3} \text {. }
$$

This relation is a first indication of the fact, that there is no on principle a difference between size effects of superclusters and size effects of clusters. However, an important quantitative difference exists: The distances between 
the centres of two neighbouring clusters in the supercluster are much greater than the distances between two neighbouring atoms in the corresponding clusters. Due to it, in superclusters not only the cluster-pair approximation of the cluster-cluster interaction energy

$$
\Delta E \approx \sum_{I<J}^{l .} E_{I J}\left(R_{I J}\right)
$$

is justified, but also the restriction to the only contributions of the nearest neighbouring pairs of clusters:

$$
E_{l J}\left(R_{l J}\right) \approx\left\{\begin{array}{rc}
E_{1}\left(R_{1}\right) & \text { for } R_{l J}=r_{2} \\
0 & \text { otherwise }
\end{array}\right.
$$

$\left(R_{I J}=\right.$ distance between the centres of the cluster $I$ and $J, r_{2}=$ nearest neighbouring distance of two clusters).

3) To verify the relations (7) and (7a), we now define a mean first coordination number, $C N_{1}(L)$, of a cluster $\mathrm{Li}_{13}$ inside of the supercluster $\left(\mathrm{Li}_{13}\right)_{L}$ as the total number of all nearest neighbouring clusters devided by number $L$. Here, for the sake of simplicity, we suppose the nearest peripheral and the nearest radial distances between two clusters $\mathrm{Li}_{13}$ of the icosahedral supercluster to be the same.

The last column of Table 2 shows, that the cluster-cluster interaction energy of one pair of interacting clusters, $E_{1}=\Delta E /\left(L \cdot C N_{1}(L)\right)$, is nearly constant for all icosahedral superclusters. That means: the formation of superclusters is simply controlled by an optimization of the first coordination of the cluster units inside of the supercluster:

$$
\Delta E / L \approx 0.5 E_{1} \cdot C N_{1}(L) .
$$

It is obvious, that equation (8) is equivalent with the equations (7) and (7a).

The validity of the relations (6) and (8) has been proved for various structures of superclusters with different relative orientations of the icosahedral units $\mathrm{Li}_{13}$ : linear chains, squared structures, simple cubes, body-centered cubes, and face-centered cubes. In every case, the description by the simple analytical relations (6) and (8) was excellent. Therefore, we conclude:

The size effect of the cluster-cluster interaction energy in superclusters is mainly a size effect of the averaged first coordination number of the cluster units. The chemical nature of the specific metal, the internal structure of the cluster units as well as the relative distances and orientations of the clusters inside of the supercluster only determine the values of the constants $a, b$, and $E_{1}$ in the Eqs. (6) and (8).

This result explains, why mass spectra of superclusters $\left(\mathrm{Au}_{13}\right)_{L}$ just show the preferred occurrence of superclusters with $L=13$ and $L=55$ cluster units $[5,6]$ : The corresponding structure of a full shell icosahedron represents a polyhedron of an optimum topology. 

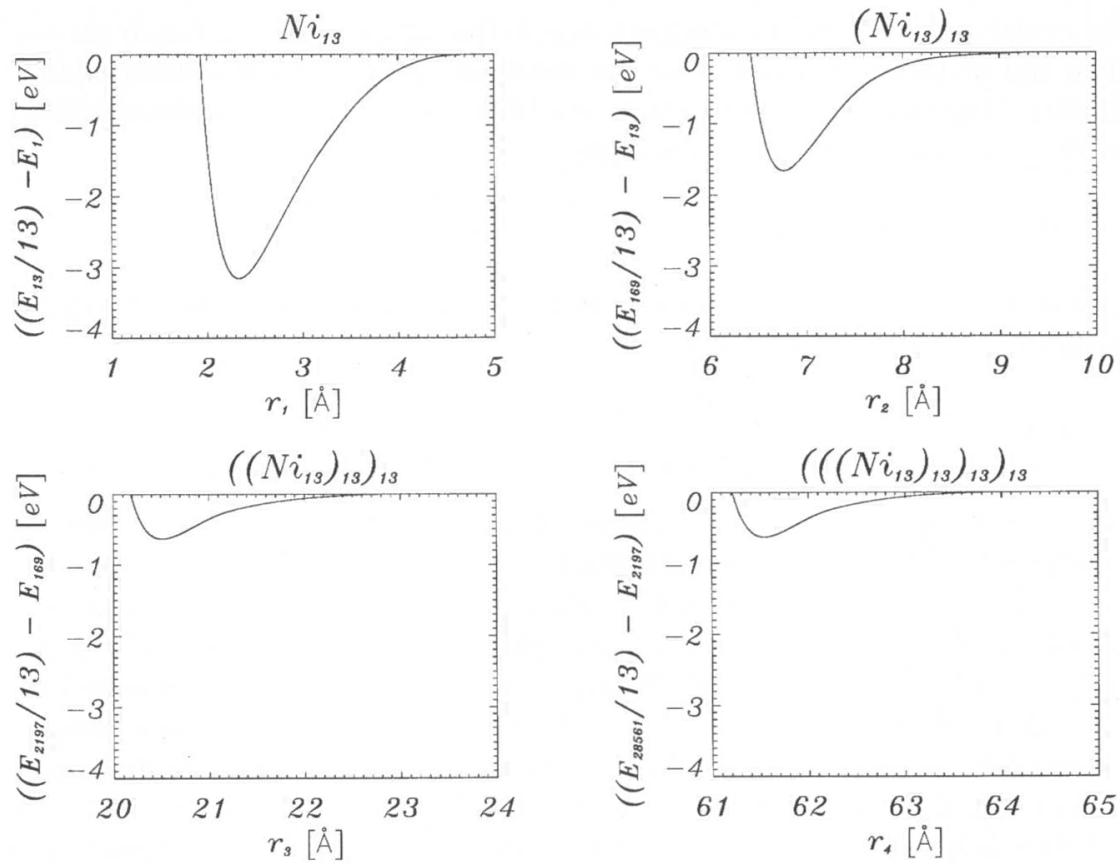

Fig. 4. Formation energies of icosahedral superclusters of nickel, $\Delta E_{k}$, for 4 steps of generation line 2 .

\subsubsection{Generation line 2}

As a second possibility, we investigate now a stepwise generation of icosahedral structures of clusters/superclusters of the 3 elements $\mathrm{Li}, \mathrm{Ni}$, and $\mathrm{Au}$ :

step 1: $13 M \rightarrow M_{13}$ (see section 3.1.)

$$
r_{1}=\text { optimum distance of } M-M
$$

step 2: $13 M_{13} \rightarrow\left(M_{13}\right)_{13}$ (see section 3.3.1 for $n=1$ )

$r_{2}=$ optimum distance of $M_{13}-M_{13}$

step 3: $13\left(M_{13}\right)_{13} \rightarrow\left(\left(M_{13}\right)_{13}\right)_{13}$

$r_{3}=$ optimum distance of $\left(M_{13}\right)_{13}-\left(M_{13}\right)_{13}$

step 4: $13\left(\left(M_{13}\right)_{13}\right)_{13} \rightarrow\left(\left(\left(M_{13}\right)_{13}\right)_{13}\right)_{13}$

$r_{4}=$ optimum distance of $\left(\left(M_{13}\right)_{13}\right)_{13}-\left(\left(M_{13}\right)_{13}\right)_{13}$.

Each step, $k$, uses the optimized product of the preceding step, $k-1$, as a rigid unit to build up the next icosahedral structure with a new optimized cluster-cluster distance $r_{k}$. 
Table 3. Equilibrium data of $k=1,2,3,4$ steps of generation of superclusters from superclusters of $\mathrm{Ni}_{13}$ (line 2): $r_{k}=$ shortest supercluster-supercluster distance, $\Delta E_{k}=$ binding energy of superclusters per supercluster-unit.

\begin{tabular}{rrl}
$k$ & $r_{k}[\AA]$ & $-\Delta E_{k}[\mathrm{eV}]$ \\
\hline 1 & 2.332 & 3.156 \\
2 & 6.764 & 1.662 \\
3 & 20.514 & 0.636 \\
4 & 61.540 & 0.636 \\
\hline
\end{tabular}

Fig. 4 presents the cluster/supercluster-formation energies of line 2

$$
\Delta E_{k}=\frac{E_{k}-13 E_{k-1}}{13} \quad \text { for } k=1,2,3,4,
$$

in dependence of the distance $r_{k}$ for the element nickel, Table 3 the corresponding values at the equilibrium.

Qualitatively, the results of lithium and gold are similar. Of course, the magnitudes of $\Delta E_{k}$ and $r_{k}$ differ from that ones of nickel.

A careful analysis of the structures of the superclusters shows a shortening of the distances between the outer contacting atoms of the interacting units of the superclusters in comparison with the constant value of $r_{1}$ : $-0.232 \AA$ in step $2,-0.010 \AA$ in step 3 , and $-0.002 \AA$ in step 4 . In the same sequence, the relative values of the cluster-cluster-formation energy, $\Delta E_{k} / \Delta E_{1}$, decreases: 0.526 in step 2, 0.201 in step 3, and 0.201 in step 4.

Obviously, the generation line 2 does not stop at step 4 . The growth of superclusters can be repeated stepwise with a constant energetic rate of growth.

\section{Properties of shell-like superclusters $\left(M_{13}\right)_{L}$}

\subsection{Cohesive energy of superclusters}

Now, we discuss the generation line 1 more generally and assume the supercluster $\left(M_{13}\right)_{L}$ to have a structure of $n$ geometrically closed shells of units $M_{13}$. Then, the number $L$ of cluster units can be expressed by a cubic polynomial of the number of shells, $n$ :

$$
L(n)=a_{3} n^{3}+a_{2} n^{2}+a_{1} n+a_{0} .
$$

The coefficients $a_{k}$ of Eq. (10) are given in [21] for 15 highly symmetrical particles.

The total energy of interaction between all units $M_{13}$ of the supercluster is

$$
\Delta E=0.5 \cdot L \cdot E_{1} \cdot C N_{1}(L) .
$$


For shell-like superclusters, also the mean $L$-dependent coordination numbers $C N_{1}(L)$ are analytical expressions of the number of shells, $n$ :

$$
C N_{1}(L(n))=2\left(b_{3} n^{3}+b_{2} n^{2}+b_{1} n+b_{0}\right) / L \text {. }
$$

The values of the coefficients $b_{i}$ are given in [21]. Thus, from the Eqs. (11) and (12) follows a polynomial representation of the total interaction energy of the supercluster

$$
\Delta E(n)=E_{1}\left(b_{3} n^{3}+b_{2} n^{2}+b_{1} n+b_{0}\right) .
$$

From the Eqs. (10) and (13), the cohesive energy of the supercluster, $\Delta E / L$, can be written as

$$
\Delta E / L=A_{0}+A_{-1} n^{-1}+A_{-2} n^{-2}+\ldots
$$

with the bulk-value of the supercluster

and

$$
(\Delta E / L)_{x}=A_{0}=\frac{E_{1} b_{3}}{a_{3}}
$$

$$
A_{-1}=\left(b_{2} a_{3}-a_{2} b_{3}\right) E_{1} /\left(a_{3}^{2}\right), \ldots
$$

\subsection{Surface dependent properties of superclusters}

We define the surface energy, $E_{s}(L)$, of a supercluster of $L$ rigid interacting basic units $M_{13}$ by the relation

$$
E_{s}(L)=\Delta E(\infty)-\Delta E(L) \text {. }
$$

Putting the Eqs. $(10,14$, and 15) into Eq. (16), we obtain

$$
E_{s}(n)=-\left[B_{2} n^{2}+B_{1} n+B_{0}+B_{-1} n^{-1}+\ldots\right],
$$

with

$$
B_{2}=a_{3} A_{-1}, B_{1}=a_{3} A_{-2}+a_{2} A_{-1}, \ldots
$$

The question, what is the surface of a supercluster, is difficult quantitatively to be answered. As a crude approximation, we assume the surface of the supercluster to be the surface of the polyhedron with faces simply constructed from the centres of the cluster units $M_{13}$ at the outer shell of the supercluster. (In reality, the surface must be greater.) Then, the surface area, $A$, follows as

$$
A=f r^{2} \text {, }
$$

with $r$ being an edge length of the polyhedron. The quantity $f$ is a form factor specific for the special polyhedron. At an edge of a supercluster of $n$ shells, $(n+1)$ cluster units $M_{13}$ are positioned with the distance $r_{2}$ between two neighbouring units. Thus, the edge length can be expressed by the shell number $n$

$$
r=(n+1) r_{2}
$$


and the surface area is a squared polynomial of $n$ :

$$
A=f r_{2}^{2}\left(n^{2}+2 n+1\right) \text {. }
$$

Now, a simple relation of the specific surface energy, $\sigma$, follows:

$$
\sigma=\frac{E_{s}}{A}=-\left[c_{0}+c_{-1} n^{-1}+c_{-2} n^{-2}+\ldots\right],
$$

with $c_{0}=\sigma(x)=B_{2} /\left(f r_{2}^{2}\right), c_{-1}=\left(B_{1}-2 B_{2}\right) /\left(f r_{2}^{2}\right), \ldots$

Similarly, analytical formulae for other mechanical properties of superclusters like the isotropic surface stress or the compressibility can be derived $[21,22]$.

\section{Concluding remarks}

Icosahedral clusters $M_{13}$ of alkaline metals, transition metals, and noble metals have diameters greater than or equal to the range of the direct interactions between two metal atoms. Our numerical calculations have shown, that such clusters interact in superclusters $\left(M_{13}\right)_{L}$ at the equilibrium by the only attraction of the nearest neighbouring pairs of the clusters. Because of the collective character of the metallic interaction considered by the nonlinear dependence of the embedding functions $F$ on the host electron density $\varrho$ (Eqs. 3 and 4 ), this result is not trivial.

For a defined structure of the supercluster, which has been determined experimentally $[1,3,5,6,9]$, the cohesive energy of the supercluster only depends on the size dependent mean coordination of a cluster by the next clusters inside of the supercluster. This result justifies a simple topological model of the cluster-cluster interaction in metallic superclusters proposed eight years ago [23].

\section{Acknowledgement}

The authors are indepted to the Deutsche Forschungsgemeinschaft and to the Fonds der Chemischen Industrie for financial support.

\section{References}

1. G. Schmid, Struct. Bonding 62 (1985) 51.

2. B. K. Teo, Polyhedron 7 (1988) 2317.

3. G. Schmid and N. Klein, Angew. Chem. 98 (1986) 910.

4. M. R. Hoare and P. Pal, J. Cryst. Growth 17 (1972) 77; Nature (London) 236 (1972) 35 .

5. H. Feld, A. Leute, D. Rading, A. Benninghoven and G. Schmid, J. Am. Chem. Soc. 112 (1990) 8166: Z. Phys. D17 (1990) 73.

6. G. Schmid, in: Clusters and Colloids, ed. by G. Schmid, Verlag Chemie, Weinheim, New York, Basel, Cambridge, Tokyo, 1994 (p. 205). 
7. H. Müller and Ch. Opitz, Z. Phys. Chem. 187 (1994) 179.

8. H. Müller and Ch. Opitz, Croat. Chem. Acta 68 (1995) 721.

9. G. Schmid, Polyhedron 7 (1988) 2321.

10. M. S. Daw and M. I. Baskes, Phys. Rev. B 29 (1984) 6443.

11. S. M. Foiles, Phys. Rev. B 32 (1985) 3409.

12. S. M. Foiles, M. I. Baskes and M. S. Daw, Phys. Rev. B 33 (1986) 7983.

13. M. S. Daw, S. M. Foiles and M. I. Baskes, Mater. Sci. Rep. 9 (1993) 251.

14. E. Clementi and C. Roetti, At. Data Nucl. Data Tables 14 (1974) 177.

15. A. D. McLean and R. S. McLean, At. Data Nucl. Data Tables 26 (1981) 197.

16. M. S. Daw, Phys. Rev. B 39 (1989) 7441.

17. B. Zhang and Y. Ouyang, Phys. Rev. B 48 (1993) 3022.

18. A. F. Voter, in: Intermetallic Compounds, Vol. 1, ed. by J. H. Westbrook, and R. L. Fleischer, John Wiley Sons Ltd, 1994, p. 77.

19. H.-G. Fritsche, Z. Phys. Chem. 191 (1995) 219.

20. B. Fehrensen, Thesis Jena, 1995.

21. H.-G. Fritsche, Z. Phys. Chem. 187 (1994) 195.

22. H. Müller, H.-G. Fritsche and L. Skala, in Clusters of Atoms and Molecules, Springer Series in Chemical Physics, Vol. 52, p. 114, Berlin, Heidelberg, New York, London, Paris, Tokyo, Hong Kong, Barcelona, Budapest (1994).

23. H.-G. Fritsche, D. Bonchev and O. Mekenyan, J. Less-Common Met. 141 (1988) 137. 